\title{
NETWORK OF ORIENTAL ROBOTIC TELESCOPES
}

François R. Querci*

Observatoire Midi-Pyrenées

14, Ave Edouard-Belin

F 31400 Toulouse Cedex, France

We should like to report on the Network of Oriental Robotic Telescopes, otherwise the ORT network. Our objective is non-stop observations of variable stars by collaboration with other networks of automated photometric telescopes, complementary to the ORT network in longitude and latitude intervals, such as the GNAT in the U.S.A., the Chilean cordillera stations, the South African stations, etc. As a first step we will deal with photometric telescopes, then we will extend them to spectroscopy.

From 12-year archives of meteorological satellites, it appeared that sites located around the north latitude from $15^{\circ}$ to $35^{\circ}$ and from $10^{\circ}$ west to $110^{\circ}$ east in longitude have high-quality astronomical conditions with a significant annual number of clear nights. Such sites involve Islamic countries from Morocco to the western deserts of China. In addition to their astronomical history, the Islamic countries are suitable because they have high mountains in semi-desert areas, i.e. a clear sky with low telluric absorption. The final site selection will be based upon local astronomical tests, such as seeing and scintillation measurements.

Meteorological prospecting together with the local access facilities ought to give a list of network sites not subjected to the same air streams. The minimum number of the network stations needed to follow variable stars each night without interruption, will be defined around 10 to 12 .

As for the photometric technique, we advocate differential photometry which, of course, implies a comparison of the flux of the variable star, $\mathrm{V}_{\mathrm{a}}$, with the flux of two (or more) comparison stars, A

*Present co-investigators: S.Kadiri CNCPRST, Rabat, Morocco, Z.Benkhaldoun, University of Marrakech, Morocco, M.Querci, OMP, Toulouse, France, H.Gashut, LCRSSS, Tripoli, Libya, K.Hussein, University of Tripoli, Lebanon, A.H.Sultan, University of Sana'a, Yemen and the engineers of OHP, France. 
and $B$, known to be non-variables and which are located in the neighbourhood of $V_{a}$. To freeze the sky transparency changes during the observation, we develop techniques allowing the three stars $\mathrm{V}_{\mathrm{a}}, \mathrm{A}$, $\mathrm{B}$ and the sky to be observed simultaneously (by using many telescopes or one large field telescope with a battery of CCD cameras). This leads to an improved accuracy and a saving of observing time by minimizing the telescope manoeuvres and by removing the need for succesive observations of the comparison stars. Simultaneous observations of the three stars reach high accuracy even when the sky conditions are slowly variable.

The final choice between the multiple-telescope technique with photomultipliers as receivers and the single large-field telescope with a battery of large CCD cameras, depends on many factors, such as the type of research projects foreseen, the technical advantages of each technique, and the relative cost of each technique.

The data collected by each station will be automatically and simultaneously transmitted by telecommunications satellites to all the scientific centres of the network, making common data reductions possible.

A design for a complete typical station: weather unit, telescopes, photometers and antenna for satellite communications, etc. has been made at the Observatoire de Haute Provence (OHP) in France. There, the engineers have analyzed the advantages and the disadvantages (reliability etc.) of each of the various possible ways of simultaneous star observations. After having tested the multiple-telescope solution with small telescopes, a 1-m diameter telescope with a large field and a battery of CCD cameras will be soon under test.

Included in the project are schemes for teaching and training in the use of the astronomical technology employed in the network, supplied by OHP, and also basic courses on astrophysics and opportunities for student practice on a fully equipped small telescope, to be given in universities of Arabic and Islamic countries that ask for them.

Scientific and technical participation will be discussed during local or regional workshops. The first is planned to be held in September 1994 in Jordan. In addition to the teaching organization, questions to be discussed will include local site-testing campaigns, cooperative scientific programmes to be developed with the network, choice of the first and second generations of auxiliary instruments (photometers, spectrographs, etc.), practical organization of the network board, 
financial support by the interested countries and international organizations, etc.

In conclusion, the ORT network, a network of robotic telescopes, will complement the automated stations located in other longitude and latitude intervals, allowing large international collaborative variable-star programmes to be run in a non-stop way for several nights or weeks.

This project is partly supported by Institut National des Sciences de l"Univers (INSU/CNRS) and by Département des Alpes de HauteProvence, where OHP is located.

Discussion: Ansari pointed out that a very good site had been found in the Indian Himalaya. D.L. Crawford (Kitt Peak) gave a brief account of his project for a worldwide network of small telescopes, which would be, in effect, an extension of Querci's proposal.

A.Boksenberg (Royal Greenwich Observatory) spoke about the facilities available on the Canary Islands, especially those wholly or partly owned by the U.K. He explained how these might be made available to African astronomers, emphasizing that, in principle, all major international installations were now open to any qualified astronomer with a programme judged to be of suitable merit. Because of great demand, however, many worthy applications were denied time. A lone astronomer from any country was unlikely to be successful, and those without experience of working on large, or even on medium-sized, instruments would be well advised to join an international group and thus share in the data obtained during an observing run. Writing an observing application that convinces convinces the time-allocation committee to award time is difficult so, again, isolated astronomers with limited experience should join a group of more experienced observers. Travel funds for astronomers from outside the U.K. were available, in special circumstances, on application through the proper channels.

Discussion: In reply to Percy, Boksenberg said that service observing is available and will soon be preferred. Kurtz emphasized that applications for observing time and travel funds could be very daunting for isolated astronomers, who need a liaison person to show them what to do. Onuora mentioned the possibility of sending a Nigerian astronomer to the Canaries to learn about solar observations. She was advised to contact Prof. F. Sanchez, Instituto de Astrofisica de Canarias. In answer to Querci, Boksenberg said that sand-wind from the Sahara had not proved to be a major problem. 